\title{
26 Projektergebnisse als freie Bildungsinhalte (OER)
}

\author{
Matthias Kunkel
}

Der Zuschlag für die Projektförderung 2012 versetzte die an optes beteiligten Hochschulen in die angenehme Lage, in den folgenden Jahren Maßnahmen für die Verbesserung der Mathematik-Kenntnisse ihrer angehenden Studierenden in den verschiedenen MINT-Fächern entwickeln und umzusetzen zu können. Die in optes zu erstellenden Materialien und Dokumente würden aber mit Sicherheit auch für andere Hochschulen, die keine entsprechende Förderung erhielten, von Interesse sein. Deshalb wurde schon im Projektantrag ein Teilprojekt „Dissemination“ festgelegt. Dessen Hauptaufgabe war es, alle Projektergebnisse interessierten Dritten bereitzustellen. ${ }^{1}$

Ebenfalls bereits im Projektantrag festgelegt wurde, dass alle erstellten Dokumente und Materialien, wie zum Beispiel Lernmodule, Testfragen oder Anleitungen, als Open Educational Resources (OER) ${ }^{2}$ unter einer Creative-Commons-Lizenz zu veröffentlichen sind. Ebenso sollten alle Softwareentwicklungen, die aus dem Projekt heraus angestoßen wurden, als Open-Source-Software unter der General Public Licence bereitgestellt werden. ${ }^{3}$ Entscheidend hierfür war die Auffassung der Antragsteller*innen, dass auch andere interessierte Hochschulen und Einrichtungen die Projektergebnisse nutzen können sollten, die ohnehin aus Steuergeldern erstellt wurden.

Die Materialien sollten aber nicht nur frei zugänglich im Sinne des OpenAccess-Ansatzes sein. Die an den Lehr- und Lerninhalten aus optes interessierten Anwender*innen sollten diese entsprechend des Konzepts der „fünf Freiheiten“4 auch an eigene Bedarf anpassen und weitergegeben dürfen.

\footnotetext{
Zum Vorgehen und zu den Aktivitäten des Teilprojekts Dissemination siehe Kapitel 28 und 29. Eingeführt wurde der Begriff Open Educational Resources im Jahr 2002 von der UNESCO (UNESCO 2002, S. 8). Der Begriff "Ressource" bezieht sich dabei nicht nur auf Inhalte, sondern umfasst ,the creation of open source software and development tools, the creation and provision of open course content, and the development of standards and licensing tools. The outputs of all three may be grouped together under the term Open Educational Resources (OER)" (UNESCO / Albright 2005, S. 1). Im deutschsprachigen Raum wird der Begriff dagegen vor allem für ,frei verwendbare Lern- und Lehrmaterialien“ (Mruck et al. 2011, S. 246) verwendet.

Siehe hierzu auch das nachfolgende Kapitel 27.

4 David Wiley beschreibt diese Freiheiten bzw. Rechte als die „5Rs of Openness (...) Retain, Reuse, Revise, Remix, Redistribute“ (Wiley 2014). Ihr Vorliegen ist ausschlaggebend dafür, dass aus Bildungsinhalten Open Educational Resources werden.
} 
Der Entscheidung, alle optes-Materialien als freie Bildungsinhalte zu veröffentlichen, lag außerdem die Hoffnung zugrunde, durch eine möglichst große Verbreitung genügend qualifiziertes Feedback zu erhalten, um diese Lerninhalte und -angebote in einem weiteren Schritt zu verbessern. Je mehr Hochschulen also optes-Materialien nutzen würden, umso mehr Verbesserungsvorschläge würden eingehen, um diese Materialien weiter zu optimieren.

\subsection{Das Lizenzmodell der Creative Commons}

Um die optes-Projektergebnisse als Open Educational Resources (OER) zu veröffentlichen, bedurfte es eines passenden Lizenzmodells. Mit der Creative Commons (CC) wurde ein Modell gewählt, das relativ einfach zu handhaben ist und inzwischen breite Akzeptanz gefunden hat. ${ }^{5}$

Creative-Commons-Lizenzen ermöglichen es, urheberrechtlich geschützte Werke wie die von optes erstellten Materialien und Inhalte, der Allgemeinheit zur freien und ungehinderten Nutzung zur Verfügung zu stellen. Die Werke unterliegen damit weiter dem Urheberrecht. Die Lizenzen räumen vertraglich aber bestimmte Nutzungsrechte ein. Und sie ,,verbessern die rechtliche Transparenz und Sicherheit sowohl für Nutzer als auch für Rechteinhaber“" (Kreutzer 2016, S. 15).

Hochschulen und andere Anwender*innen, die optes-Angebote nutzen und verändern wollen, haben somit eine sichere Rechtsgrundlage, ohne für jede Modifikation um Erlaubnis bei den Urheber*innen nachfragen zu müssen. Durch diese Rechtssicherheit erhöhte sich prinzipiell die Chance, dass die optes-Materialien im Lehralltag anderer Hochschulen auch tatsächlich zum Einsatz kommen.

Anders als beim herkömmlichen Urheberrecht ${ }^{6}$ mit seinem Prinzip des „Alle Rechte vorbehalten“ räumen Open-Content-Lizenzen wie die Creative Commons (CC) die freie Nutzung unter definierten Bedingungen ein. Diese Nutzungsbedingungen spiegeln sich in den Namen der jeweiligen CC-Lizenzen wieder. Durch die Kombination dieser Bedingungen ergibt sich eine Auswahl von insgesamt sechs verschiedenen CC-Lizenzen für urheberrechtlich geschützte Werke. ${ }^{7}$

5 In der Zwischenzeit haben sich die Creative Commons (CC) als die Open-Content-Lizenzen etabliert. Till Kreutzer konstatiert 2016: „Aufgrund seiner Popularität und weiten Verbreitung kann man CC heute de facto als Standard für Open-Content-Lizenzen bezeichnen“ (Kreutzer 2016, S. $30)$.

6 Einen guten Überblick über die im deutschen Rechtssystem gültigen Urheber- und Verwertungsrechte bietet Thomas Hartmann (Hartmann 2014, S. 25ff.).

7 Darüber hinaus bietet Creative Commons noch die Deklaration CC0, mit der eigene Werke gemeinfrei erklärt werden können: https://creativecommons.org/publicdomain/zero/1.0/legalcode [10.06.2020]. 


\section{BY Namensnennung 8}

Diese CC-Lizenz fordert nur die Nennung der Autor*innen des Werkes, die Quelle, Rechteinhaber*innen und die Lizenz. Weitergehende Beschränkungen für die Nutzer*innen bzw. Lizenznehmer*innen gibt es nicht. Das Werk kann also beliebig bearbeitet und auch zur kommerziellen Nutzung weitergegeben werden. Die CC-BY-Lizenz ist die einzige CC-Lizenz, die mit allen anderen fünf CC-Lizenzen kombiniert werden kann.

\section{BY-SA Namensnennung-Share Alike ${ }^{9}$}

Diese Lizenz verlangt neben den Hinweispflichten auf die Autor*innen, die Quelle, Rechteinhaber*innen und die Lizenz, dass das Werk unter der gleichen Lizenz weitergegeben wird (Share Alike). Dies gilt auch für neu erstellte Werke, die mit CC BY-SA lizensiertes Material wiederverwenden.

\section{BY-ND Namensnennung-Keine Bearbeitungen ${ }^{10}$}

Diese Lizenz gestattet keine Bearbeitungen des Werkes. Die Weitergabe ist dagegen uneingeschränkt gestattet. Auch eine kommerzielle Nutzung ist erlaubt. Wie bei allen anderen CC-Lizenzen besteht aber die Pflicht, die Autor*innen, die Quelle, Rechteinhaber*innen und die Lizenz zu nennen.

\section{BY-NC Namensnennung-Nicht kommerziell ${ }^{11}$}

Werke unter dieser Lizenz können ohne Einschränkungen bearbeitet und verändert werden. Die Weitergabe ist erlaubt, nicht aber eine kommerzielle Nutzung. Hierunter fällt nicht nur der Verkauf oder die gebührenpflichtige Nutzung des Werkes an sich, sondern auch die Bereitstellung im Rahmen eines kostenpflichtigen Angebots wie zum Beispiel einer kommerziellen Weiterbildung. ${ }^{12}$

\section{BY-NC-SA Namensnennung-Nicht kommerziell-Share Alike ${ }^{13}$}

Diese Lizenz gestattet Bearbeitungen des Werkes. Dessen Veröffentlichung muss aber wieder mit der gleichen Lizenz erfolgen. Werden Bearbeitung vorgenommen, ist der Bearbeiter*innen verpflichtet, auf die Übernahme des Ursprungswerkes und die hieran vorgenommenen Änderungen hinzuweisen. Eine kommerzielle Nutzung ist nicht gestattet.

\footnotetext{
Lizenztext siehe: https://creativecommons.org/licenses/by/4.0/legalcode.de [10.06.2020].

Lizenztext siehe: https://creativecommons.org/licenses/by-sa/4.0/legalcode.de [10.06.2020].

Lizenztext siehe: https://creativecommons.org/licenses/by-nd/4.0/legalcode.de [10.06.2020].

Lizenztext siehe: https://creativecommons.org/licenses/by-nc/4.0/legalcode.de [10.06.2020].

12 Eine eindrückliche Übersicht, wer überhaupt nur NC-lizensierte Inhalte nutzen darf und in welchen Fällen, findet sich bei Till Kreutzer (Kreutzer 2016, S. 51).

13 Lizenztext siehe: https://creativecommons.org/licenses/by-nc-sa/4.0/legalcode.de [10.06.2020].
} 


\section{BY-NC-ND Namensnennung-Nicht kommerziell-Keine Bearbeitungen ${ }^{14}$}

Diese ist die restriktivste Variante der CC-Lizenzen und bietet kaum Freiheiten gegenüber dem traditionellen Urheberrecht. Die Lizenz schließt sowohl die kommerzielle Nutzung als auch Bearbeitungen vollständig aus. Es bestehen Hinweispflichten auf den Autor*innen, die Quelle, Rechteinhaber*innen und die Lizenz.

\subsection{Auswahl der Creative-Commons-Lizenzen für optes}

Zu Beginn des optes-Projekts 2012 lagen die CC-Lizenzen in der Version 3 vor. Für das Projekt wurden zunächst die portierten, ins Deutsche übersetzten und an die deutsche Rechtsordnung angepassten Fassungen genutzt. Ab 2018 kamen auch die Lizenzen der internationalen Version 4 für neu veröffentlichte Materialien zum Einsatz.

Dass die optes-Projektergebnisse unter Creative-Commons-Lizenzen veröffentlicht werden sollten, stand seit dem Projektantrag fest. Aber welche der sechs möglichen CC-Lizenzen für die Nutzung im optes-Projekt in Frage kamen, musste in der ersten Projektphase noch mit allen Projektbeteiligten geklärt werden. Deren Zahl hatte sich mit dem Projektstart gegenüber der Antragstellung erheblich vergrößert. Und eine Entscheidung über die zu nutzenden Lizenzen sollte unbedingt einvernehmlich erfolgen.

$\mathrm{Zu}$ Beginn diskutiert wurde die Frage, ob eine kommerzielle Nutzung der optes-Lerninhalte und -Materialien ausgeschlossen sein sollte. Einige Projektbeteiligte befürchteten, dass Verlage möglicherweise die optes-Materialien ohne Einschränkung in ihren kommerziellen Angeboten wiederverwenden könnten. Mit den Lizenzen CC BY-NC, CC BY-NC-ND und CC BY-NC-SA hätten entsprechende Lizenzen zur Verfügung gestanden, die eine solche Nutzung untersagten.

Das Risiko einer kommerziellen Verwertung durch Dritte wurde letztlich aber als zu gering angesehen. Schon die Veröffentlichung der Materialien unter einer CC BY-SA würde eine solche Form der Nutzung verhindern. Denn bei einer Aufbereitung der optes-Materialien für ein kommerzielles Verlagsangebot hätte dieses gemäß des Share-Alike-Prinzips ebenfalls wieder unter eine CC-BY-SA-Lizenz gestellt werden müssen. Und damit wäre auch die kommerzielle Weiterverwendung dieses Angebots durch andere erlaubt. Dieser lizenzrechtliche Umstand würde die Wiederverwendung der optes-Angebote für kommerzielle Anbieter damit letztlich wenig attraktiv machen.

Zudem wäre es für das optes-Projekt praktisch unmöglich gewesen, bei einer Veröffentlichung der Materialien unter einer NC-Lizenz etwaige Verletzungen dieser effektiv zu verfolgen und zu ahnden. Dies ist aber zwingend notwendig, wenn Materialien eine kommerzielle Nutzung verwehrt werden soll. Till Kreutzer

14 Lizenztext siehe: https://creativecommons.org/licenses/by-nc-nd/4.0/legalcode.de [10.06.2020]. 
stellt hierzu fest: „Ist der Lizenzgeber ... nicht bereit oder in der Lage, durch rechtliche Schritte gegen potentielle Verletzungen der NC-Einschränkungen vorzugehen, macht es von vornherein kaum Sinn, sie aufzuerlegen“" (Kreutzer 2016, S 52).

Durch die prinzipielle Erlaubnis der kommerziellen Nutzung konnten optesMaterialien auch Anwender*innen zur Verfügung gestellt werden, die diese Materialien im Rahmen von kostenpflichtigen Ausbildungs-, Weiterbildungs- und Schulungsangeboten einsetzen möchten. Hierunter fallen zum Beispiel auch private Schulen und Hochschulen. Einige private Bildungseinrichtungen haben im Verlauf des Projekts dann auch in der Tat optes-Materialien heruntergeladen und konnten diese dank der gewählten CC-Lizenz rechtmäßig nutzen.

Da die relativ uneingeschränkte CC-BY-Lizenz für einige Projektbeteiligte zu viele Freiheiten für die Nutzung durch Dritte ermöglichte ${ }^{15}$ und damit zu viel Kontrolle über die selbst erstellten Werke abgegeben werden musste, blieben für die Veröffentlichung der in optes erstellten Materialien und Inhalte letztlich zwei der sechs CC-Lizenzen übrig.

Lerninhalte, Übungsmaterialien, Handreichungen und Anleitungen, die Dritte weiterentwickeln und an eigene Bedürfnisse anpassen können sollten, wurden unter die Lizenz CC BY-SA gestellt. Damit würden künftige Nutzer*innen den größtmöglichen Grad an Freiheit bei der Bearbeitung und Weitergabe der Materialien haben. Zugleich stellt die Lizenz sicher, dass mögliche Bearbeitungen durch Dritte ebenfalls mit der gleichen Lizenz wie die ursprünglichen optes-Materialien weitergegeben und entsprechend genutzt werden.

Für Dokumente, an denen Dritte ohne explizite Genehmigung keine Veränderungen vornehmen sollten, kam in optes als zweite CC-Lizenz die CC BY-ND zum Einsatz. Diese war für die Veröffentlichung bestimmter Projekt- und Evaluationsberichte vorgesehen, aus denen gemäß wissenschaftlicher Praxis zitiert werden konnte. Gegenüber dem normalen Urheberrecht erlaubte diese Lizenz aber noch die Weitergabe der entsprechend lizensierten Werke.

In der Praxis wurden der ganz überwiegende Teil der in optes bereitgestellten Projektergebnisse unter der Lizenz CC BY-SA veröffentlicht. Zum Stichtag 31.3.2020 waren auf optes.de nur fünf Dokumente mit der CC BY-ND ausgezeichnet - gegenüber 238 Dokumenten mit der großzügigeren CC BY-SA-Lizenz.

15 Dieser Umstand macht sie gerade zur bevorzugten Lizenz in OER-Kreisen, da nur die CC-BYLizenz mit allen anderen CC-Lizenzen kombinierbar ist (Creative Commons 2009). 


\subsection{Umsetzung im Projektalltag}

Nach der Entscheidung im Projektteam für die beiden Lizenzen CC BY-SA und CC BY-ND mussten im nächsten Schritt die Voraussetzungen geschaffen werden, um diese Lizenzen in der Praxis zu nutzen. Zunächst erfolgte die Konfiguration der von optes verwendeten Lernplattform ILIAS. Dazu wurden nur die beiden unterstützten CC-Lizenzen in der zentralen Metadaten-Verwaltung von ILIAS aktiviert. Schon bei der Erstellung der Lernmodule, Fragenpools, Glossare, Dateien und Kurse konnten Autor*innen die gewünschte Lizenz per Checkbox auswählen und die Inhalte entsprechend auszeichnen. Bei diesem Schritt erfolgte auch die Nennung der Autor*innen.

In der ILIAS-Lernplattform wird die jeweils gewählte Lizenz auf der Info-Seite des jeweiligen Lerninhalts oder Dokumentes angezeigt. Bei Materialien, die als Exportdatei für den Download durch Dritte bereitgestellt werden, erhält auch die Download-Datei eine Angabe über die gewählte Lizenz in ihren Metadaten. Ab 2019 konnten entsprechend ausgezeichnete Dateien zusätzlich mit dem sogenannten OER-Harvester von ILIAS automatisch identifiziert und an zentraler Stelle auf der optes-Plattform angezeigt werden.

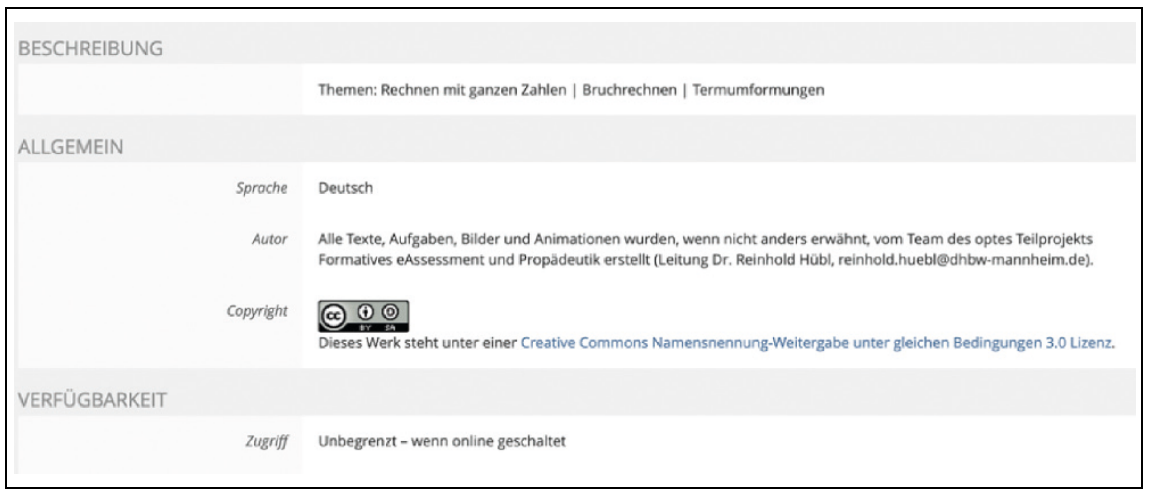

Abbildung 1: Info-Seite eines in ILIAS erstellten Vorkurses mit Hinweis auf CC-Lizenz

Um bei allen Projektbeteiligten ein gemeinsames Verständnis und einen Mindeststand an Kenntnissen über CC-Lizenzen zu schaffen, wurde schon in der ersten Förderphase ein gemeinsamer Workshop im Rahmen eines Quartalstreffens veranstaltet. Die auch im Team-Wiki bereitgestellten Informationen zu den Lizenzen und ihren Implikationen wurden hier noch einmal für alle Beteiligten ausgiebig vorgestellt und diskutiert. Ebenso wurde die Handhabung von Materialien Dritter besprochen und ein gemeinsames und einheitliches Vorgehen beschlossen.

Für die Auszeichnung der Materialien mit der entsprechenden CC-Lizenz waren in optes immer die Autor*innen der jeweiligen Teilprojekte zuständig. Diese 
mussten auch überprüfen und sicherstellen, dass alle verwendeten Inhalte für eine Auszeichnung mit einer CC-Lizenz geeignet waren. Vor der Veröffentlichung von Projektergebnissen überprüfte das Teilprojekt Dissemination die entsprechenden Angebote auf das Vorhandensein der entsprechenden Metadaten-Angaben und ergänzte sie gegebenenfalls.

Für die Öffentlichkeit bereitgestellt wurden die optes-Materialien von Anfang an im optes-Anwendernetzwerk ${ }^{16}$. Dieses ist ein eigener Bereich innerhalb des Internetauftritts des optes-Projekts. Alle interessierten Anwender*innen können sich für das Anwendernetzwerk selbst und unverbindlich anmelden. Mit der Registrierung wird jede*r Benutzer*in automatisch Mitglied in einer Gruppe auf optes.de. In dieser Gruppe befinden sich unter anderem ein Diskussionsforum für Fragen rund um die Angebote und der mit einem Wiki umgesetzte Materialienpool, in dem Angebote beschrieben werden und der jeweilige Download-Link bereitgestellt wird.

Der anonyme Zugang zu den optes-Materialien wurde im Projektteam diskutiert, aber aus mehreren Gründen verworfen. Zum einen bestand der Wunsch nach dem Aufbau einer Community von optes-Anwender*innen und der Möglichkeit, einfach mit diesen Personen in Kontakt zu treten. Wären alle Materialien ohne Anmeldung herunterladbar gewesen, hätte die Projektbeteiligten nicht erfahren, wer sich an welcher Hochschule konkret für ihre bereitgestellten Angebote interessiert und eine Übernahme dieser Inhalte in die eigene Lehre erwägt. Zum anderen entstand mit dem Beitritt zum Anwendernetzwerk und der Aufnahme in die zugehörige Gruppe automatisch eine Mailingliste, über die alle Interessierten außerhalb des optes-Projekts einfach und fortlaufend über Neuerungen und aktualisierte Angebote informiert werden konnten.

Außer auf dem optes-Portal werden ausgewählte optes-Materialien inzwischen auch im Zentralen Repositorium für Open Educational Resources der Hochschulen in Baden-Württemberg (ZOERR) ${ }^{17}$ bereitgestellt. Der Zugriff auf die optes-Materialien erfolgt hier ohne Registrierung im optes-Anwendernetzwerk. Service-Angebote, wie die Benachrichtigung der Anwender*innen über Neuerungen, sind bei der Bereitstellung über die Landesrepositorien entsprechend nicht mehr möglich. Eine Bereitstellung von Materialien über diese Kanäle erhöht aber die Sichtbarkeit von optes deutlich, womit das Projekt und seine Projektergebnisse auch bei neuen Anwendergruppen bekannt wird. Da die von optes verwendeten CC-Lizenzen eine Weitergabe der Werke nicht einschränken, können optes-Materialien inzwischen möglicherweise auch noch auf anderen Webseiten zur Verfügung stehen.

Die Entscheidung, alle Projektergebnisse von optes als freie Bildungsinhalte der Allgemeinheit zur Verfügung zu stellen, hat sich für das Projekt als richtig erwiesen. Mit den CC-Lizenzen wurde eine rechtliche Grundlage gewählt, die für Projektteilnehmer*innen und externe Anwender*innen Rechtssicherheit bietet und

16 Mehr zum Aufbau und zur Funktion des Anwendernetzwerks findet sich in Kapitel 29.

17 Siehe https://www.oerbw.de [10.06.2020]. 
klar regelt, was mit den Materialien gemacht werden kann. Ohne die Entscheidung für eine Veröffentlichung als OER hätte das Ziel der Dissemination, dass auch Projektexterne optes-Angebote nutzen, kaum erreicht werden können.

Trotz der Konkurrenz durch andere Propädeutik-Angebote im Bereich MINT, die während der Projektlaufzeit entstanden ist, setzten zu Jahresbeginn 2020 bereits 15 Hochschulen und neun Schulen optes-Materialien ein, um entsprechende Unterstützungsangebote im Bereich der Mathematik bereitstellen zu können. Hierzu gehören auch einige Hochschulen, die gar nicht ILIAS als zentrales Lernmanagementsystem nutzen, die in optes bereitgestellten Fragenkataloge aber in ihr LMS überführt haben.

Dagegen ist die Anzahl an qualifizierten Rückmeldungen auf die Angebote und Vorschläge zu deren Verbesserung - anders als erwartet - relativ niedrig geblieben. Die meisten externen Anwender*innen nutzen einzelne optes-Angebote wie zum Beispiel Lernmodule oder Fragenpools als Ausgangsmaterial und passen sie an ihre Bedarfe an. Feedback an das Projekt geben sie hingegen selten.

In den Fällen aber, in denen qualifizierte Rückmeldungen von Hochschulanwender*innen an die zuständigen Teilprojekte gegeben wurden und von diesen in die optes-Angebote eingearbeitet werden konnten, hat die Qualität dieser Materialien sehr profitiert. Eine solche Form der kollaborativen Qualitätssicherung, wie sie zum Beispiel auch aus Open-Source-Projekten bekannt ist, wäre für künftige Projekte mit einem vergleichbaren Konzept für die Produktion und Dissemination von Lernangeboten sicherlich vielversprechend.

\section{Literatur}

Albright, P. (2005). Final forum report. In International Institute for Educational Planning. Internet Discussion Forum. Open Educational Resources Open Content for Higher Education, Vol. 24. Verfügbar unter http://www.ifap.ru/library/book064.pdf [20.05.2020].

Creative Commons (2009). Remixing OER: A Guide to License Compatibility. ccLearn Explanations, October 2009. Verfügbar unter https://core.ac.uk/reader/71342743 [10.06.2020].

Hartmann, T. (2014). Urheberrecht in der Bildungspraxis. Leitfaden für Lehrende und Bildungseinrichtungen. Bielefeld: W. Bertelsmann Verlag.

Kreutzer, T. (2016). Open Content - Ein Praxisleitfaden zur Nutzung von Creative-Commons-Lizenzen. Verfügbar unter http://agentur59.de/blog/wp-content/uploads/ 2015/10/Open_Content_-_Ein_Praxisleitfaden_zur_Nutzung_von_Creative-CommonsLizenzen.pdf $[\overline{1} 0.06 .20 \overline{2} 0 \overline{]}$.

Mruck, K., Mey, G. Purgathofer, P., Schön, S. \& Apostolopoulos, N. (2011). Offener Zugang. Open Access, Open Educational Resources und Urheberrecht. In M. Ebner \& S. Schön (Hrsg.), L3T Lehrbuch für Lernen und Lehren mit Technologien (S. 243-248). Berlin: epubli.

UNESCO (2002). Forum on the Impact of Open Courseware for Higher Education in Developing Countries. Final report. Verfügbar unter https://unesdoc.unesco. org/ark:/48223/pf0000128515 [10.06.2020]. 
Wiley, D. (2014): The Access Compromise and the 5th R. Beitrag im Blog "Iterating Toward Openness" vom 5.3.2014. Verfügbar unter https://opencontent.org/ blog/archives/3221 [10.06.2020].

Open Access Dieses Kapitel wird unter der Creative Commons Namensnennung 4.0 International Lizenz (http://creativecommons.org/licenses/by/4.0/deed. de) veröffentlicht, welche die Nutzung, Vervielfältigung, Bearbeitung, Verbreitung und Wiedergabe in jeglichem Medium und Format erlaubt, sofern Sie den/ die ursprünglichen Autor(en) und die Quelle ordnungsgemäß nennen, einen Link zur Creative Commons Lizenz beifügen und angeben, ob Änderungen vorgenommen wurden.

Die in diesem Kapitel enthaltenen Bilder und sonstiges Drittmaterial unterliegen ebenfalls der genannten Creative Commons Lizenz, sofern sich aus der Abbildungslegende nichts anderes ergibt. Sofern das betreffende Material nicht unter der genannten Creative Commons Lizenz steht und die betreffende Handlung nicht nach gesetzlichen Vorschriften erlaubt ist, ist für die oben aufgeführten Weiterverwendungen des Materials die Einwilligung des jeweiligen Rechteinhabers einzuholen.

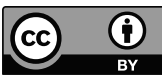

